\title{
Validation of circulating biomarkers for breast cancer screening
}

Ana Pedro ${ }^{1}$

1 University of Hull

To validate a circulating biomarker or biomarkers and the respective test kit for breast cancer screening we need at least 1000 patient samples from each primary breast cancer subtype and 1000 control samples. For the biomarker or biomarkers to be validated we need an accuracy, precision, specificity, sensitivity of $99 \%$ and a statistical power of 0.95 